\title{
NOVEL HIGHLY SENSITIVE ELISA TO MEASURE FREE, BIOACTIVE, HUMAN SOLUBLE RANKL
}

Andreas Breitwieser ${ }^{1}$, Andreea Suciu ${ }^{1}$

(1) Biomarker Design Forschungs GmbH, Vienna, Austria, A.Breitwieser@bmdf.at

INTRODUCTION: RANKL, the receptor activator of nuclear factor kappa B ligand, is an essential factor for the formation of mature osteoclasts. Together with its receptor RANK and its antagonist Osteoprotegerin (OPG) RANKL is a key regulator in bone metabolism (1). RANKL is a membrane-bound protein that can be segregated to a soluble form (SRANKL), whereas only the latter has been reported to be bioactive (2). Due to its low circulating levels and the nature of the analyte binding to OPG, free sRANKL has been proven difficult to measure: the accuracy of sRANKL measurement is compromised by the very low or undetectable levels, as observed in some patient cohorts (3). Hence, our aim was to develop a highly sensitive and specific assay that enables the direct measurement of free, bioactive, soluble RANKL in serum and plasma samples.

METHODS: The ELISA developed is based on a sandwich type format, whereas advantage of the high affinity and specificity proteinprotein interaction between SRANKL and OPG has been taken. Immobilized, recombinant OPG has been used to capture free SRANKL, which subsequently is detected with a biotin labeled anti-sRANKL antibody.

RESULTS: The data presented here, demonstrate that all samples $(n=127)$ from an unselected clinical and two apparently healthy populations had detectable free sRANKL values (Fig. 2) within the calibration range of the assay ( $0-2$ pmol/, Fig. 1). The median of serum samples, prepared immediately after blood collection and stored at $-25^{\circ} \mathrm{C}$ until measurement, was $0.14 \mathrm{pmol} / \mathrm{l}$ or $0.16 \mathrm{pmol} / \mathrm{I}$ respectively (Fig. 2, healthy cohort I + II), with a lower limit of quantification (LLOQ) of $0.01 \mathrm{pmol} / \mathrm{I}$. Assay characteristics, such as spike/recovery (Fig. 3), dilution linearity (Fig. 4) and analyte stability in whole blood (Fig. 5) as well as intra/inter-assay precision (Fig. 6) have been analysed and are presented here:

\section{Typical standard curve}

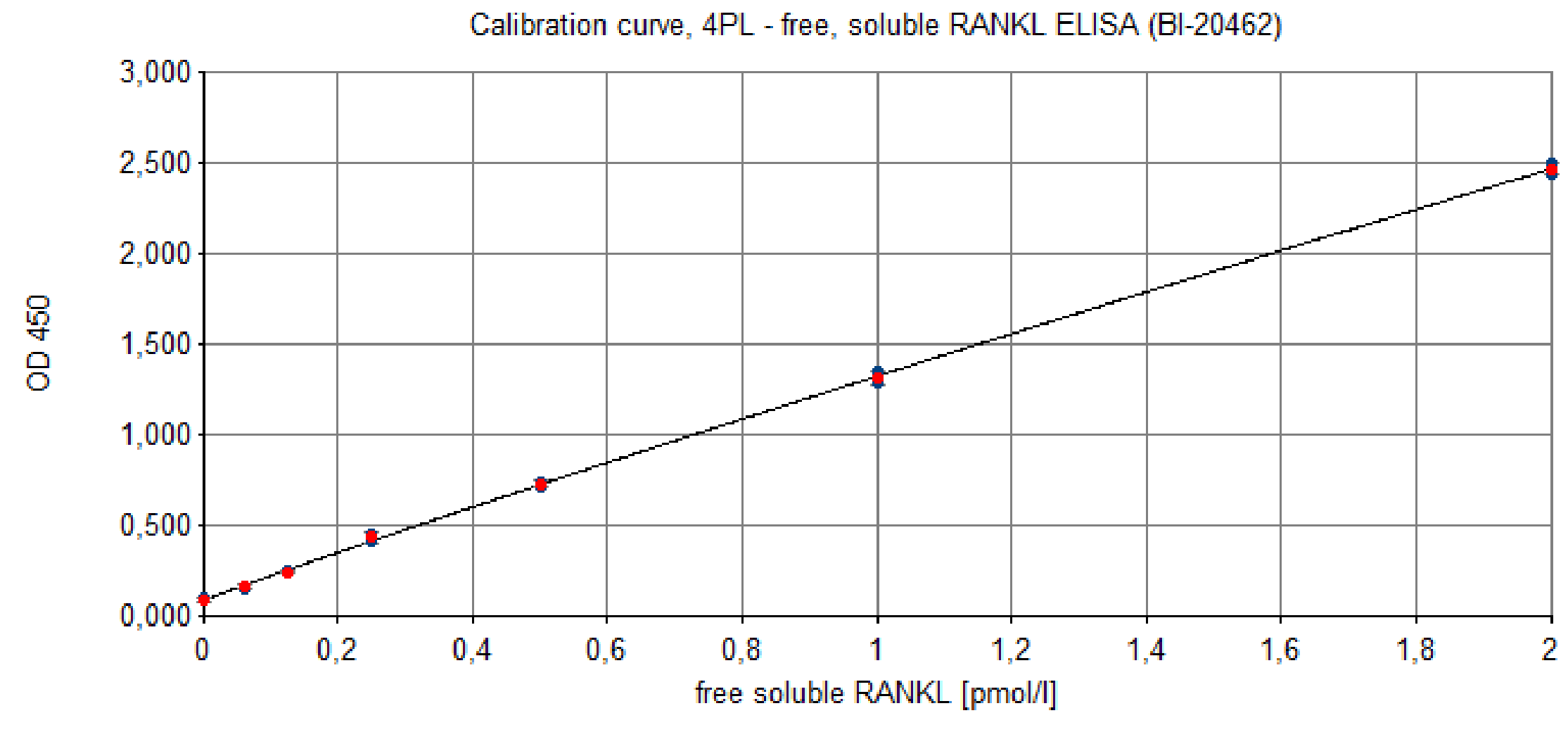

Fig. 1: Assay calibration curve - R
serum to a range of 0 - 2 pmol/l.

\section{Spike/Recovery}

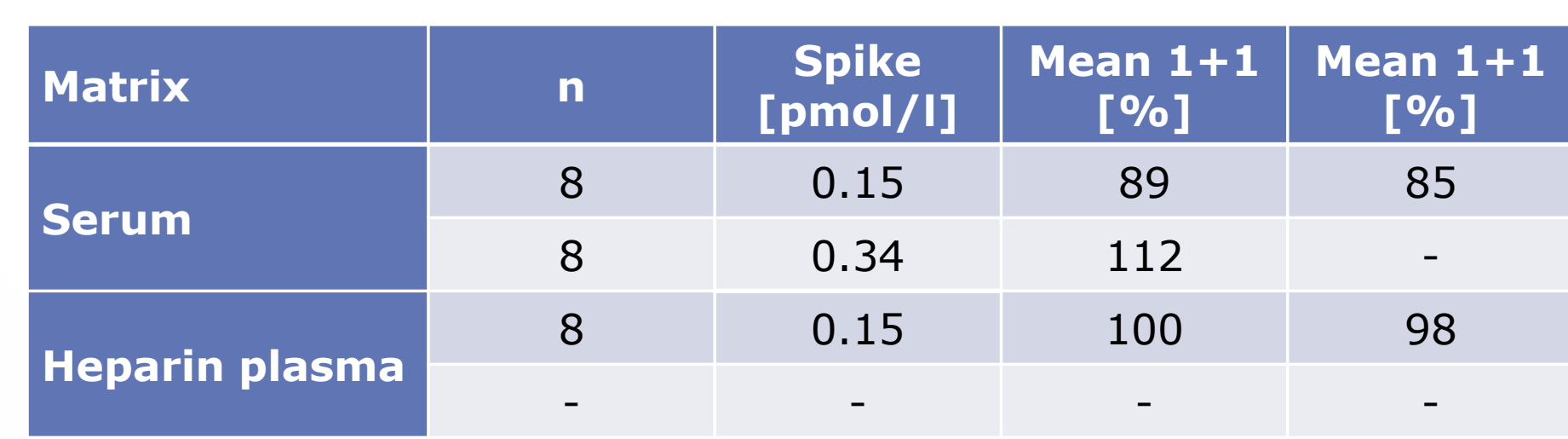

Fig. 3: Endogenous spike/recovery data in serum/heparin samples

\section{Precision}

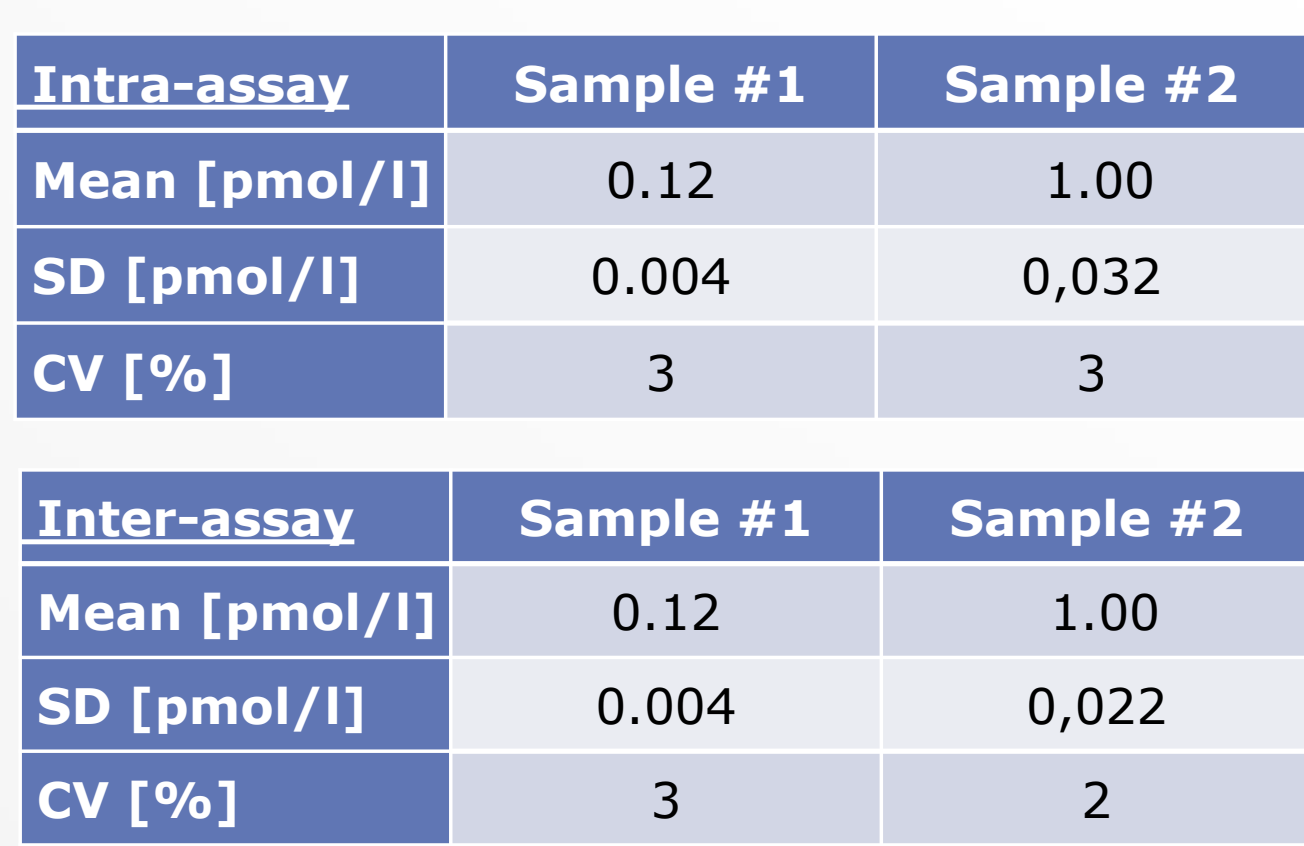

Fig. 6: Intra-/Inter-assay precision data

\section{Samples}
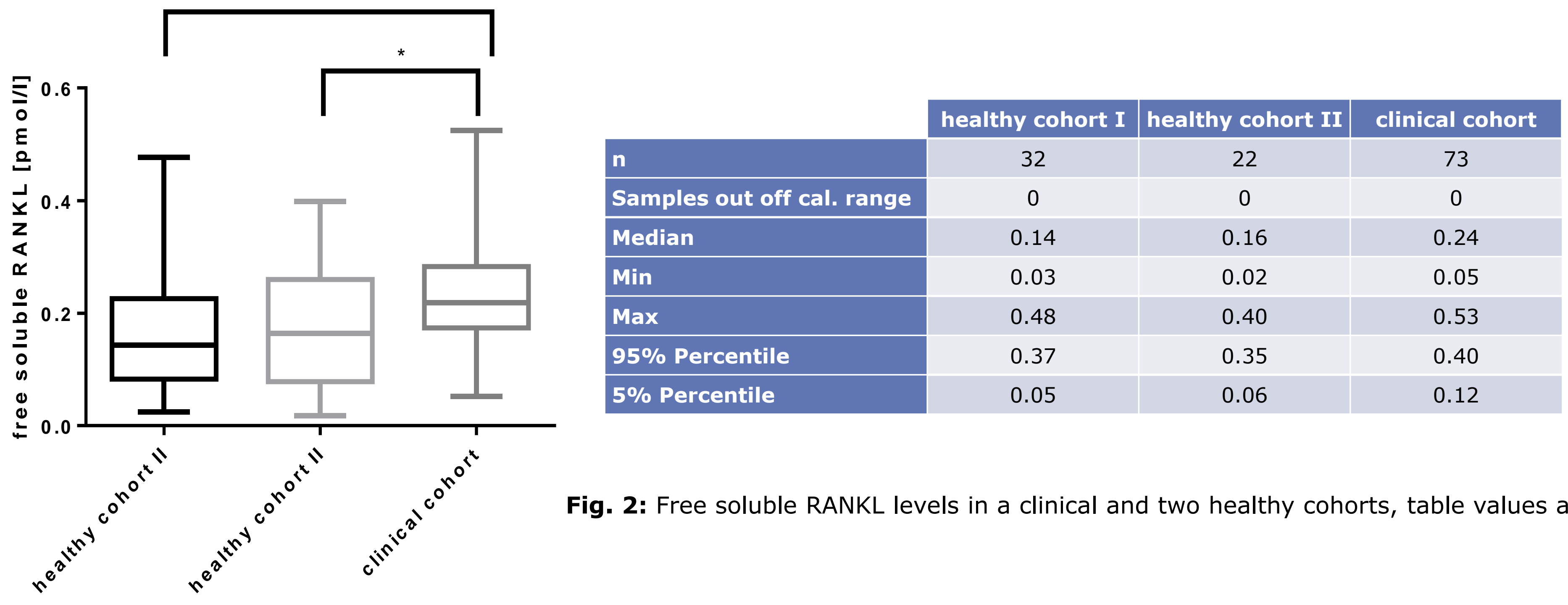

\section{Dilution Linearity}
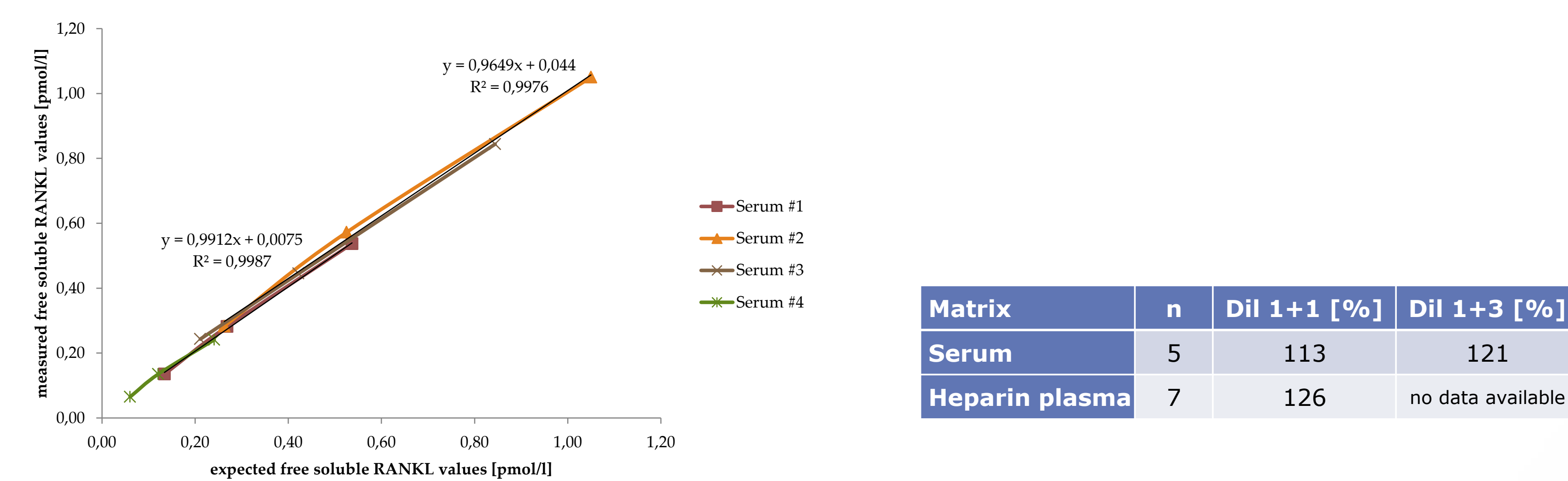

Fig. 4: Dilution linearity of serum samples - serum samples were diluted with a very low measuring serum

\section{Analyte stability}

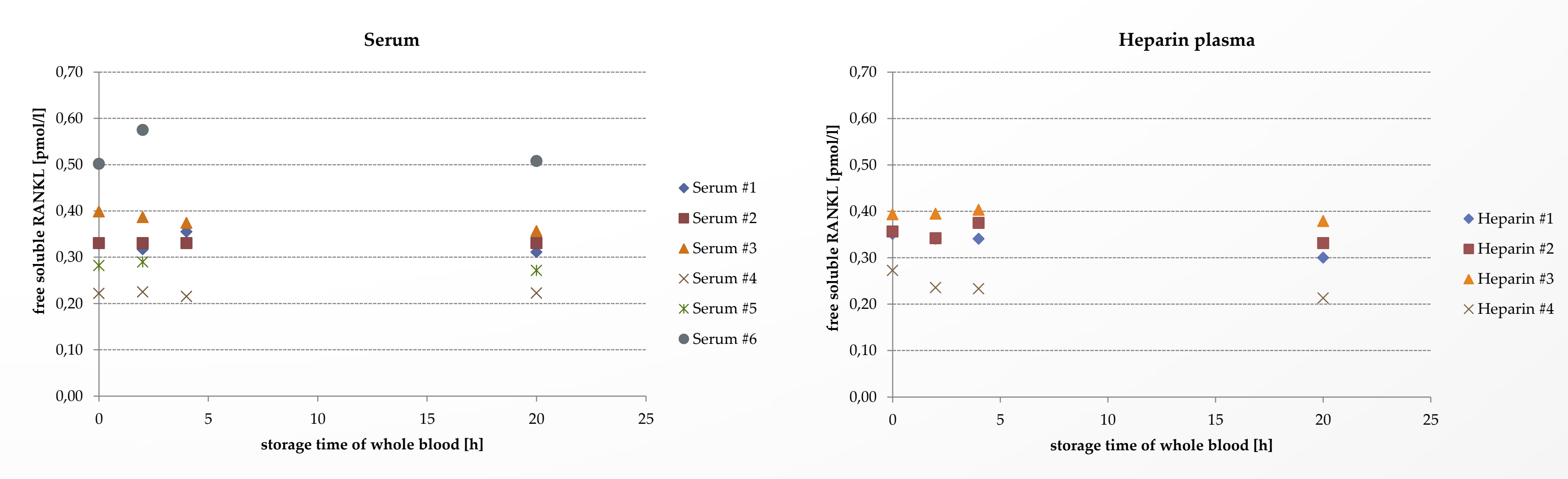

Fig. 5: Analyte stability in whole blood - whole blood was stored 0/2/4/20h in serum/heparin tubes prior to serum/heparin

CONCLUSIONS: Our novel ELISA provides a reliable and accurate tool for the quantitative determination of free, soluble, bioactive RANKL in human samples and could help to understand the underlying regulatory mechanisms of various diseases such as rheumatoid arthritis, inflammatory bowel disease, multiple myeloma, and cardiovascular disease.

(1) Biology of the TRANCE axis.

Walsh MC and Choi Y, Cytokine Growth Factor Rev. 2003; 14(3-4):251-63. (2) Orchiectomy upregulates free soluble RANKL in bone marrow of aged rats. Proell V. et al., Bone 2009; 45(4):677-81.
(3) Comparative effect of zoledronic acid versus denosumab on serum sclerostin and dickkopf-1 levels of naive postmenopausal women with low bone mass: Anastasilakis A. et al, J. Clin. Endocrinol. Metab. 2013; 98(8):3206-12. 